Technical Progress Report

\title{
PLASMA CONFINEMENT THEORY AND TRANSPORT SIMULATION
}

David W. Ross

Fusion Research Center. The University of Texas al Austin, Austin, Texas 78712

May 1, 1991 - April 30, 1992

U. S. Department of Energy, Office of Energy Research, Grant DE-FG05-88ER53266

\section{SUMMARY OF PROGRESS}

The objectives are: (1) to advance the transport studies of tokamaks, inciuding development and maintenance of the Magnetic Fusion Energy Database, and (2) to provide theoretical interpretation, modeling and equilibrium and stability studies for TEXT-Upgrade. Recent reports, publications, and conference presentations of the Fusion Research Certer are listed on attached pages [1-45].

\section{A. Magnetic Fusion Energy Database}

The MFE profile database now contains almost 1000 discharges from 6 different tokarnaks. In the current period, emphasis has heen on the development of tools to access and manipulate the data $[18,28,40,44]$. The database has been moved to an IBM/RS6000 which allows the data to be accessed via an $X$-window application. There is also a batch-like procedure using Netlib-hased soltware [28], which allows a user to mail an SQL query across the Internet and have the results mailed back to the user.

The most recent and powerful solution to the acess pro lem is a new interface, MDI. which uses Mathematica $\mathbb{B}$ as a front end [18,44]. MDI is a Mathematica package that defines a basic set of MFE-database aceess functions. The package will also accept standard SQL queries. Each function returns Mathematica-style lists, which can then be manipulated with any of the Matherratica functions. MDI also provides utility functions for plotting and analyzing the dala, essentially making the MFE dalabase an extension of Mathematica. The user may use any of the many Mathemarica tront-ends including telnet, X-Windows, or a notsbook. The MDI.m package may he obtained by anonymous FTP from the MFE-database site or by use of netmfe [28], an E-mail database interface. MDI has also been installed on a workstation at NERSC.

MDI is a example of distribuled computing. Behind the user interface, MDI calls an RPCl client program that communicates with an RPC server on the MFE-database computer. It relies on the network-communication capabilities of Mathematica to connect the user to a workstation running the Matheinatica kernal. The Marhematica kernel is then connected to the MFE-database host workstalion by a client/server pair of RPC processes. If the Mathematica kernel is to be run on the user's machine, the R.PC client program must also be obtained and installed. The MDI-RPC server is also available for users who would like to provide their own client software. The server returns ASCII tables from standard queries and may be accessed and processed by any program on the internet that has access to RPC services. 
In order to make it easier for the various laboratories to contribute data, the MFEdatabase project has been willing to accept ASCIl-coded data in almost any format. Reformatting this data for insertion into the MFE database has required writing a separate program to parse each type of tile. For example, the output lile from the OneTwo code consists of a licader, several lines of comments, severat NAMELISTs, diagnostic messages, several tables, and summary information. We are developing a tool kil which accepts, as input, descriptions of the Onc'Two formats and builds a parser [40]. The parser can then operate on the OneTwo input files and directly enter the data inio the database. This tool kit is being constructed using the tools lex and yacc.?

In the coming period we will focus our efforts on diangthening contacts with experimentalists on the various machines, obtaining additional data, and exploring statistical analysis methods.

I yohn R. Corbin, "The Art of Distributed Applications", Springer-Verlag, New York, 1990.

2'Tony Mason and Doug Brown, "lex \& yacce", Datie Dougherty, ed., O'reilly \& Associates, 1990.

\section{B. Support for TEXT-Upgrate and Diagnostics}

We have adapted an iron-core versica of the equilibrium code EFIT 3,4 to run on the IBM RS6(0)(0) [3.3]. It was decided to use only public-domain or very easily available software for mathematical subroutines and plotting packages. Preliminary studies, without plots, show that a typical case (only one time slice) can run in 10 to 20 seconds of CPU time on the RS60(0), compared to 2 or 3 minutes on the VAX 86()() . This could allow the use of EFIT for magnetic analysis in between shots.

We have designed and assembled an apparatus for the measurement of the magneticfield profile on TEXT-U [19], utilizing successive charge-exchange collisions, as suggested by Valanju. Expected capabilities are $1 \mathrm{~cm}$ spatial resolution, $\left(0.1^{\circ}\right.$ angular resolution of magnetic field direction and $1 \mathrm{~ms}$ time resolution. Tests of calibration and sensitivities are in progress. Additional theoretical work has led to a design for a multi-chord apparatus which can measure a q-profile in a single shot [8].

We have collaborated in a general review of TEXT fluctuation measurements and transport interpretation [1.3]. Work is continuing on possible attenuation effects in heavy-ion beam-probe lluctuation measurements for various plasma conditions on TEXT [21]. A simulation code was used to determine the altentation effect on broad-band fluctuation amplitudes, coherence lengths, and wave numbers. Simulation results and experimental data were compared. The analysis is being extended to other plasma conditions such as modulatedECH 'non-local' phenomena, sawteeth, and MHD. We also hope to have direct measurements of primary-beam attenuation effects from TEXT-U. For low density plasmas, attenuation effects do not seem to be a significant part of the measured thuctuation signals for any plasma condition other than MHD.

${ }^{3}$ L. L. Lio, H. St. John, and R. D. Stambaugh et all. Nuct. Fusion 25, 1611 (1985)

${ }^{4}$ E. R. Solino, G. H. Neilson, and L. L. Lio, Nucl. Fusion 301107 (1990). 


\section{Instabilities and Transport}

\section{Oscillating gas puff}

A perturbation analysis has heen developed which closely couples measurements of oscillating density, temperature, and lluctuation levels with a theoretical model in order to calculate perturbed particle fluxes $[16.29]$. Perturbation data on TEXT yielded a Mux, calculated using a simplified dissipative-trapped-electron (dte) model, which did not agree with the directly measured tlux. The lack of inward steady-state flux terms in the model may contribute substantially to this disagreement. We also investigated inodulated gas puffing using the transport code CHAPO with generic aromalous models that retain the symmetry properties of the quasilinear transport matrix in $\nabla n$ and $\nabla T$, hut allow for various dependences on plasma parameters [30]. Comparing the amplitudes and phases of the experimental perturbed fluxes, densities, temperatures, etc., with those of the simulation, we attempted to determine the critical features of the model. In particular, in analogy with diffusion-convection models, we asked whether the off-diagonal coefticients must be negative. The results were ambiguous, however, owing to the uncertainty in the particle source in the plasma core at the low plasma densities considered.

Most recently, we have the analysis to higher density cases where the effect of the source is reduced [42]. First results, where we still employed the die scaling, did not reproduce the measured profiles. Further study of the source question is needed.

\section{Edge physics and L-H transition}

In collaboration with TEXT experimentalists, with the IFS, and with General Atomics, we are pursuing a broad range of edge physics and H-mode studies.

We have calculated impurity tlow velocities in the presence of collisionality and charge exchange [2()]. We lind that ions and impurities move together in the paristel direction. In the perpencicular direction, collisional friction is insufficient to damp their independent diamagnetic motions. The resulting poloidal impurity llow can be opposite to the ion diamagnetic llow. Poloidal and toroidal rotation rates of light impurities have been measured in TEXT using Doppler shift techniques and have been compared with this theory. Besides fueling a tokamak plasma, cold, stationary neutrals near the wall can cause drag on rotating ions via charge-12xchange [37]. In addition, charge-exchange produces hot, penetrating neutrals that can enhance plasma viscosity and thermal conductivity, and can diminish ion diamagnetic drift [1()]. A cold ion [6] population is also produced that causes further drag while being heated via Coulomb collisions with hot ions. We derive analytical expressions for the drag, viscosity, thermal conductivily and plasma rotation protiles by solving a four-population driftkinetic model with physical boundary conditions at the wall. The resulting isotopic dependence of energy confinernent and the effects of varying plasma profiles near the wall have been studied.

Particle, thermal, and impurity transport in high and low density He discharges were studied and compared with the transport in $\mathrm{H}$ and $\mathrm{D}$ discharges [31]. Profiles of $T_{e}, T_{i}, n_{e}, v_{\phi}$ $v_{\theta}$ and $P_{\text {rad }}$ were measured along with the imptrity confinement time and the particle source distribution. A l-d transport simulation was used to infer local values tor the transport

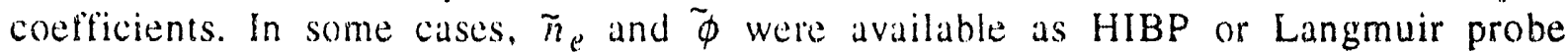


measurements. When combined with the transport results, these allow an exarnination of the importance of proposed turbulence drive mechanisms including ionization drive and radiation drive.

Traditionally neoclassical transport assumes a poloidally constant poloidal magnetic: field. Near an X-point, however, the poloidal tield is very small, so the tield lines linger at that poloidal location. We have shown this to modify particle orbits, enhancing grad B drifts [4.3]. In particular, banana orbits that bounce near the $X$-point have "square tips", due to the grad B drift acting on the particle lor a longer time. Computational and analytical calculations of particle orbits allow an evaluation of the importance of this effect (the drifts are proportional to $\checkmark$ thermal, so hotter ions are more strongly affected). Realistic poloidal fields are used to compute particle orbits. A study of changes in Plirsch-Schlüter collisional transport due to the $\mathrm{X}$-point has also been initiated.

Steady state solutions have been found for a set of equations describing the transport of particles and energy in a tokamak plasma [221. A model of uansport by turbulence is used in which the turbulence is assumed to be suppressed by shear in the equilibrium ExB flow. The equilibrium radial electric ficld which drives this flow is determined hy radial ion pressure balance. An energy source is used which represents central heating by an unspecified mechanism. The particle source is given in terms of the neutral density which is calculated from a simplified neutral transport equation. The density and temperature profiles are of two types, depending on the particle and energy somce strengths. For a axed particle source strength, they are: (1) profiles with small gradients, when the heating power is below a threshold value, and (2) profiles with large gradients near the plasma edge, when the heating power is abo"e this threshold value. The particle and energy confinement times, as functions of the heating power, are discontinuous at the power theshold, suggesting comparisons with experimental results for the L- to H-mode transition. The time dependent hehavior of the same proposed transport model exhihits confinement hifucation [35]. The hifurcation is due to the nonlinear nature of the proposed transport coerticients. Here, the equations are extended into the time domain, and nurrerical solutions are presented which illustrate the nonlinear behavior.

Effects of ionization on the linear drift wave instability is generally complicaled, because the equilibrium assumed in a generic slab model (the spatial variation of the plasma density only in the radial direction, and zero ladial particle flow) cannot be achieved owing to the presence of ionization $[12,36 \mid$. We have considered several equilibrium models, inciuding a quasi-equilibrium state, wherein the bulk plasma density evolves slowly compared to the characteristic time scule of the mode frequencies. In this case an analytical solution is obtained for the collisionless drift wave; the contribution from ionization to the temporal evolution of the spatial eigenmode is algebraic rather than exponential.

\section{Other transport studies}

We have collaborated with the IFS in ion-temperature-gradient mode simulation studies that compare transport predictions with experiment [14]. This has proved to be a useful application of the MFE database.

In addition, we are beginning to henefit from work carried out in the former Soviet Union and continued at FRC. This includes, first, a new transport mechanism appearing specifically under eyclotron wave healing of plasma in toroidal devices [41]. This mechanism 
can explain the deterioration of particle confinement in the resonant zone and other experimentally observed phenomena that accompany electron cyclotron heating. The driving force for the new transport process is the poloidally asymmetric electrostatic potential. This potential arises due to the enrichment of the population of suprathermal banana electrons bouncing at the resonance position.

Secondly, a new approach to plasma transport is described where the toroidal drift is considered as a perturbation to the motion of particles in electromagnetic oscillations, $u_{d} \ll \tilde{v}$ [45]. From the analysis of particle trajectories it is shown that there is a small group of particles that move in the direction of the toroidal drift, even if $u_{d} \ll \tilde{v}$. This small group of particles also plays a major role in anomalous transpont processes. Percolation theory is used to determine the scaling of the diffusion coefficient, $D$, which is larger than the neoclassical platea coefficient, $D p l$, in both banana and plateau regimes: $D \sim D^{p l}\left(\tilde{v} / u_{d}\right)^{4 / 7} \gg D^{p l}$. Numerical simulation results support the theoretical scaling arguments.

\section{E. Alfuén Waves}

We have investigaled heating of tokamak plasmas by toroidal Allvén eigenmodes and toroidicity-modified global Alfven cigenmodes $[25,39]$. In tokamak plasmas, the Alfven wave specturn is strongly affected by the vartiation of the equilibrium magnetic field along the major radius, giving rise, for example, to the toroidal Alfven eigenmodes (or gap modes). These modes may be viewed as resulting from the coupling of cylindrical poloidal harmonics. We have modified a previously constructed antenna-driven cylindrical code, ${ }^{5}$ which takes into account electron inertia and Landau damping and finite ion Larmor radius, to study this numerically. Assuming realistic profiles, we have investigated the toroidicity-induced Alfven eigenmodes as well as the effects of toroidicity on the cylindrical global eigenmodes. These are identified as resonances in the impedance $Z(\omega)$. We plot the wave electric field, energy deposition, energy how, and currene drive as functions of minor radius.

5D. W. Ross, Gi. L. ('hen, and S. M. Mahajan, Phys. IHuids 25, 6.52 (1982). 


\section{REPORTS AND PUBLICATIONS 1991.92}

Names underlined are those supported by the contract.

\section{publication status update:}

1. H. L. Berk, S. M. Mahajan, and Y. Z. Zhang, $m=1$ kink mode for layer widith comparable to the ion Larmor radius, Phys. Fluids B2, 351 (1.991),

2. R. L. Hickok, P. M. Schoch, T. P. Crowley, J. Heard, K. W. Gentle, D. W. Ross, A. J. Wootton, B. Richards, W. L. Rowan, D. L. Brower, N. C. Luhmann, W. A. Peebles, and C. X. Yu, Turbulent Fluctuations and Transport in TEXT, in Plasma Physics and Controlled Nuclear Fusion 1990)(IAEA, Vienna, 1991), Vol. 1, p. 229.

3. M. Kotschenreuther, H. L. Berk, R. Denton, S. Hamaguchi, W. Horton, C.-B. Kim, M. Lebrun, P. Lyster, S. Mahajan, W. H. Miner, P. J. Morrison, D. W, Ross, R. D. Sydora, T. Tajima, J. B. Taylor, P.M. Villaniu, H. V. Wong, S. Y. Xiao, and Y.-Z. Zhang. Novel Computational Methods to Predict Transport in Confinement Devices and Application to Ion Temperature Gradieni Driven Turbulence, in Plasma Physics and Controlled Nuclear. Fusion Research 1990)(IAEA, Vienna, 1991), Vol. 2, p. 361.

4. D.W. Ross, On Standard Forms for Transport Equations and Quasilinear Fluxes, Plasma Phys, and Contr. Fusion 34, 137 (1992).

5. D.W. Ross, R. V. Bravenec, C. P. Ritz, M. L. Sloan, J. R. Thompson, A. J. Wootton, P. M. Schoch, J. W. Heard, T. P. Crowley, R. L. Hickok, V. Simcic, D. L. Brower, W. A. Peebles, and N. C. Luhmann Jr., Comparison of Drift Wave Models with Flactuation Data from the Interior of the TEXT Tokamak, Phys. Fluids B3, 2251 (1991).

6. A. A. Ware, R. D. Hazeltine, M. Calvin, P. Valanju, and E Solano, The Effect of Low Energy Ions and Neutrals on Tokamak Transport, in Plasma Physics and Controlled Nuclear Fusion 1990 (IAEA, Vienna, 1991), Vol. 2, p. 361.

7. D. W. Ross, M. L. Sloan, A. J. Wootton, P. M. Schoch, J. W. Heard, T. P. Crowley, R. L. Hickok, and V. Simcic, Effect of Beam Attenuation Modulation on Fluctuation Me'asurements by Heavy Ion Beam Probe, The University of Texas Report FRCR \#380, 1990, to be published in Rev. Sci. Instrum.

8. P. M. Valaniu, Design of a Compact Multichord Detector for Plasma Curient and Ion Temperature Profiles Measurements using a Diagnostic Neural Beam. The University of Texas Report FRCR \#386, March, 1991.

9. L C. Wiley, A UNIX Device Driver for a TransLink II Transputer Board, The University of Texas Report FRCR \#383, January, 1991.

additional reports and pablications

10. R. D. Hazeltine, M. D. Calvin, P. M. Valanju, and E. R. Solano, Analytical Calculation of Neutral Transport and its Effect on Ions, Nucl. Fusion 32, 3 (1992).

11. Y.Z. Zhang and S. M. Mahajan, On Broken Ballooning Symmetry, Phys. Lett. A 157, $133(1991)$.

12. Y, Z, Zhang and S. M. Mahajan, Impurity and Neutral Effects on the Dissipative Drift Wave in Tokamak Eage Plasmas, Phys. Fluids 4, $2(17$ (1992). 
13. R. V. Bravenec, K. W. Gentle, B. Richards, D.W Ross D. C. Sing, A, J. Wootton, D. L. Brower, N. C. Luhmann Jr., W. A. Peebles, C. X. Yu, T. P. Crowley, J. W. Heard, R. L. Hickok, and P. M. Schoch, Core Turbusence and Trunsport Studies on the TEXT Tokamak, to be published in Phys. Fluids (1992).

14. M. Kotschenreuther, H, V. Wong, P. L. Lyster, H. L. Berk, R. Denton, W, H. Miner, and $\mathrm{P}$. Valanju, Comparisons of Theortically Predicted Transport from Ion Temperatare Gradient Instabilities to L-Mode Tokamak Experiments, University of Texas Repont IFSR No. 532, 1991, submitted to Phys. Rev. Letters.

15. S. M. Mahajan, P. M. Valaniu, and W. L. Rowan, Form Invariance in ConvectiveDiffusive Systems with Applications to Impurity Transport, to be published in Phys. Fluids (1992).

16. B. Richards, M. E. Austin, R. V. Bravenec, D. L. Brower, K. W. Gentle, R. L. Hickok, N. C. Luhmann, A. Ouroua, W. A. Peebles, P. E. Phillips, D. W. Ross, W. L. Rowan, P. M. Schoch, P. M. Valaniu, J. C. Wiley, A. J. Wootton, X. Z. Yang, and C. X. Yu, Tests of Plasma Transport Models via Perturbation Techniques, to be published in Nucl. Fusion, 1992.

17. P, M Valaniu, M. D. Calvin, R. D. Hazeltine, and E. R. Solano, The Effect of Charge Exchange on Plasma Flows, The University of Texas Report IFRS $\# 540,1992$, to be published in Phys. Fluids (1992).

18. J.C. Wiley, W. H. Miner, Ji., and D.W. Ross, MDI: Mathematica Database Interface for the MFEDB. The University of Texas Report FRCR \#411, 1992.

American Physical Society, Division of Plasma Physics, November, 1991:

19. R. D. Berigtson, P. M. Valaniu, M. F. Muldoon, and D. H. Bluestein II, Status of a qdiagnostic on TEXT-U, Bull. Am. Phys. Soc. 36, (1991).

20. M. D. Calvin, R. D. Hazeltine, A. G. Meigs, W. L. Ruwan, E. R, Solano, and P. M. Vilanju, Effect of Collisional Friction on lmpurity Flow, Bull. Am. Phys. Soc. 36 , (1991).

21. J. W. Heard, T. P. Crowley, P. M. Schoch, R. L. Hickok Jr., and D. W. Ross, Attenuation Effects on Heavy Ion Beam Probe Broalband Fluctuation Wave. Number Mecasurements on TEXT, Bull. Am. Phys. Soc. 36, (1991).

22. F. L. Hinton and $\perp$. W. Wilev, Particle and Energy Confin:ment Bifurcation in Tokamaks, Bull. Am. Phys. Soc. 36, (1991).

23. W. Horton, W. Rowan, J.C. Wiley, J. Q. Dong, and Y. Karzhavin, Impurity Transport in Tokamaks, Bull. Am. Phys. Soc. 36, (1991).

24. Y. Y. Karzhavin and P., . Valiniu, Analysis of plasma parameters in regimes with high and low impurity confinement under Ohmic heating, Bull. Am. Phys. Soc. 36, (1991).

25. W. Q. Li, S. M. Mahajan, D.W. Ross, and M. Chu, Kinetic Description of Toroidal Alfuén Eigenmodes, Bull. Am. Phys. Soc. 36. (1991).

26. S. M. Mahajan and Y.Z. Zhang, Broken Ballooning Symmetry and 2-D Drift Wave Eigenmode in Tokamaks, Bull. Am. Phys. Soc. 36, (1991).

27. R. T.McCinn. Variational Determination of Optimal MHD Equilibria, Bull. Am. Phys. Soc. 36, (1991). 
28. W. H. Miner, Jr., R. T. McCann, and J. C. Wiley, MFE Database Data Manipulation Tools, Bull. Am. Phys. Soc. 36, (1991).

29. B. Richards, M. E. Austin, R. V. Bravenec, D. L. Brower, K. W. Gentle, R. L. Hickok, N. C. Luhmann, W. A. Peehles, P. E. Phillips, D. W. Ross W. L. Rowan, P. M. Schoch, and P.M. Valaniu, Analysis of Plasma Perturbation Experiments Using Theoretical Models, Bull. Am. Phys. Soc. 36, (1991).

30. D. W. Ross L.C. Wiley, B. Richards, and R. V. Bravenec, Gas Puffing and Anomalous Transport Theory, Bull. Am. Phys. Soc. 36, (1991).

31. W. L. Rowan, A. G. Meigs, M. E. Austin, R. L. Hickok, B. Richards, C. P. Ritz, P. M. Schoch, P. M. Valanju, B. Z. Zhang, and Z. M. Zhang, Transpori in Helium Discharges in the Texas Experimental Tokamak, Bull. Am. Phys. Soc. 36, (1991).

32. A. Sen, W. Q. Li, and S. M. Mahajan, 2-D Equilibrium of a Plasma in the VlasovMaxwell Systems, Bull. Am. Phys. Soc. 36, (1991).

33. E. R. Solano, EFIT in a Fast Computer, Bull. Am. Phys. Soc. 36, (1991).

34. P. M. Valanju, S. M. Mahajan, and W. L. Rowan, Form Invariance in ConvectiveDiffusive Systems with Applications to Impurity Transport, Bull. Am. Phys. Soc. 36. (1991).

35. I. C. Wiley and F. Hinton, Time Dependent Behavior of a Particle and Energy Confinement Bifurcation Model, Bull. Am. Phys. Soc. 36, (1991).

36. Y, Z.Zhang and S. M. Mahajan, Insestigation of the lonization Effects on the Drift Wave Instability, Bull. Am. Phys. Soc. 36, (199i).

presented at the Sherwood Theory Meeting, Santa Fe, 6.8 April, 1992:

37. M. D. Calvin, R. D. Hazeltine, and P. M. Valanju. Effects of Neutrals on Tokamak Eage Plasma, paper 1C4().

38. E. G. Heo, B. H. Park, D.-I. Choi, J. Hernandex, W. Horton, and P. N, Yushmanov, Turbulent Ion Transport in the General Toroidal Geometry, paper 1C11.

39. W. Q. Li, S. M. Mahajan, D. W. Ross, and M. Chu, Kinetic Description of Toroidal Alfvén Eigenmodes, paper $2 \mathrm{C5}$

40. R. T. McCinn, W. H. Miner, Jr., and J. C. Wiley, Using LEX and YACC to Build a Parser for the MFEDB, paper 3C25.

41. V. I. Pozniak and P. N. Yushmanov, Specific Transport Processes under ECRH in Tokamaks, paper 2C9.

42. D. W. Ross, B. Richaids, W. L. Rowan, and J,C. Wiley Transport Simulation of Oscillating Gas-Puff on TEXT using Theoretical Models, paper 2C10.

43. E. R. Solano and R. D. Hazeltine. X-point effects on particle orbits and neoclassical transport, paper IC 30 ).

44. L.C. Wiley and W. H. Miner, Jr., MDI: Mathematica Database Interface for the MFE Database, paper 2C.32.

45. P N Yushmanov, Neoclassical Diffusion in a Turbulent Plasma, paper 1D15. 

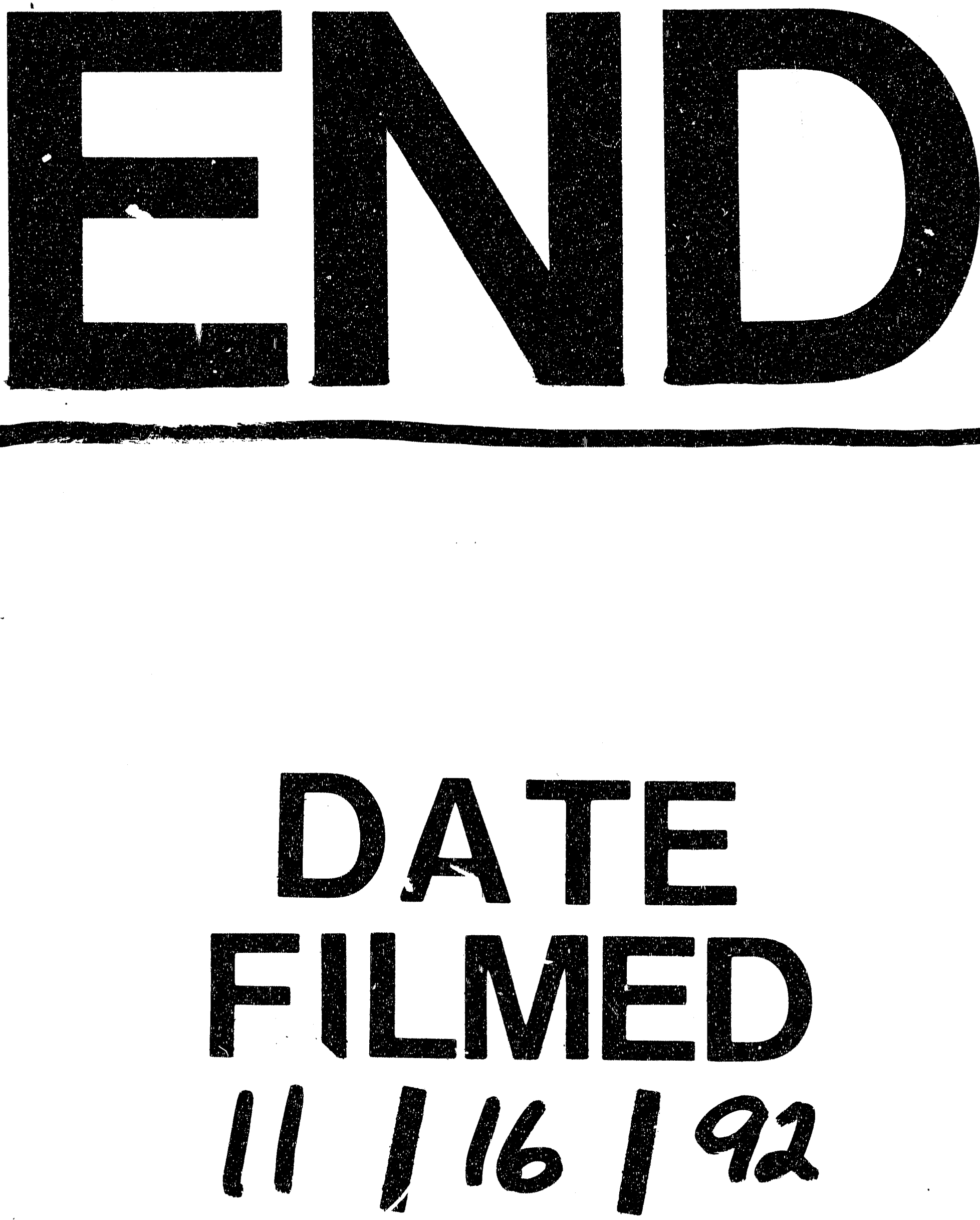


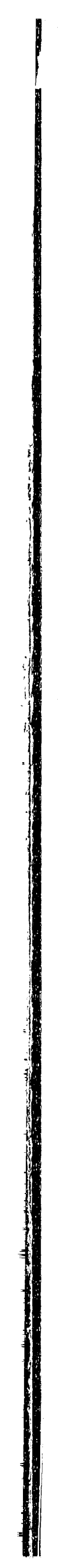

